Article

\title{
Variation of Triterpenic Acids in 12 Wild Syzygium formosum and Anti-Inflammation Activity on Human Keratinocyte HaCaT
}

\author{
Hyun-ah Park ${ }^{1, \dagger}{ }^{\dagger}$ Mi Yoon Kim ${ }^{2,3, \dagger}$, Nan-Young Lee ${ }^{1}$, Jaeyoon Lim ${ }^{4}$, Kyu-been Park ${ }^{1}$, Chang-Kyu Lee ${ }^{1}$, \\ Van Dao Nguyen ${ }^{5}$, Jaehan Kim ${ }^{4}{ }^{(}$, Jong-Tae Park ${ }^{1,6, *}$ (1) and Jong-Il Park ${ }^{2,3, *}$ \\ 1 CARBOEXPERT Inc., Daejeon 34134, Korea; hapark@carboexpert.com (H.-a.P.); \\ nylee@carboexpert.com (N.-Y.L.); kbpark@carboexpert.com (K.-b.P.); cklee@carboexpert.com (C.-K.L.) \\ 2 Department of Biochemistry, College of Medicine, Chungnam National University, Daejeon 35015, Korea; \\ miyoonkim@cnu.ac.kr \\ 3 Translational Immunology Institute, Chungnam National University, Daejeon 35015, Korea \\ 4 Department of Food Science and Nutrition, Chungnam National University, Daejeon 34134, Korea; \\ daebakch9086@gmail.com (J.L.); jaykim@cnu.ac.kr (J.K.) \\ 5 Biotechnology Faculty, Binh Duong University, Thu Dau Mot 820000, Vietnam; nvdaohou@gmail.com \\ 6 Department of Food Science and Technology, Chungnam National University, Daejeon 34134, Korea \\ * Correspondence: jtpark@cnu.ac.kr (J.-T.P.); jipark@cnu.ac.kr (J.-I.P.) \\ + These authors contributed equally.
}

check for

updates

Citation: Park, H.-a.; Kim, M.Y.; Lee, N.-Y.; Lim, J.; Park, K.-b.; Lee, C.-K.; Nguyen, V.D.; Kim, J.; Park, J.-T.; Park, J.-I. Variation of Triterpenic Acids in 12 Wild Syzygium formosum and Anti-Inflammation Activity on Human Keratinocyte HaCaT. Plants 2021, 10, 2428. https://doi.org/ $10.3390 /$ plants10112428

Academic Editor: Cinzia Sanna

Received: 6 October 2021

Accepted: 8 November 2021

Published: 10 November 2021

Publisher's Note: MDPI stays neutral with regard to jurisdictional claims in published maps and institutional affiliations.

Copyright: (c) 2021 by the authors. Licensee MDPI, Basel, Switzerland. This article is an open access article distributed under the terms and conditions of the Creative Commons Attribution (CC BY) license (https:/ / creativecommons.org/licenses/by/ $4.0 /)$.

\begin{abstract}
Syzygium formosum (Wall.) Masam leaf is known as a Vietnamese traditional herbal medicine used to treat atopic dermatitis and stomach ulcers. Recently, its potent anti-allergic effects were reported with possible active compounds analysis. Here, we collected S. formosum leaves from 12 wild trees and compared compositions of triterpenic acids (TA) with Centella asiatica. Antiinflammatory activities of S. formosum leaf extract (SFLE) was compared with C. asiatica extract (CAE) using human keratinocyte, HaCaT. In this study, up to seven TAs were identified in SFLE, while only madecassic and asiatic acids were detected in the CAE. Total TA content varied among SFLE, but asiatic, corosolic, and betulinic acids were the major components. Surprisingly, wild tree sample 12 (S12) contained total TA of $27.2 \mathrm{mg} / \mathrm{g}$ dry-leaves that was 5-fold greater than that in the C. asiatica sample, and S4 had the highest content of asiatic acid ( $12.6 \mathrm{mg} / \mathrm{g}$ dry-leaves) that accounted for $50 \%$ of the total TA. S4 and S12 showed more than 3-fold higher anti-oxidative power than the CAE. In the UVB irradiation model, S4 and S12 $(5 \mu \mathrm{g} / \mathrm{mL})$ strongly repressed mRNA levels of pro-inflammatory cytokines (IL-1 $\beta$, IL-6, and IL-8) and COX-2, while the CAE at the same condition showed moderate or weak repression. The difference in anti-inflammation effects between the SFLE and the CAE was also confirmed by protein quantifications. Taken together, SFLE has great potentials as a new cosmeceutical ingredient with a higher amount of skin-active phytochemicals.
\end{abstract}

Keywords: anti-inflammation; secondary metabolite; skin cell; Syzygium formosum; triterpenic acid

\section{Introduction}

Plant extracts are common source for the bioactive compounds that have been used in traditional remedies for human diseases. Medicinal herbs are developed in different countries and consumed as teas or can be applied on the disordered skin area [1,2]. Among them, Korean ginseng is one of the most popular plant used by ingestion, while Centella asiatica extract is the most successful active ingredient for cosmetics. Aloe vera and Camellia genus are also well-known functional plant for topical uses. Those plants have been intensively investigated for the identification of active phytochemicals and evaluations of biological activities.

C. asiatica extract contains various triterpene derivatives such as asiatic acid, madeccassic acid, asiaticosides, and madecasosides [3,4]. Those triterpene derivatives are regarded as hallmarks of functional phytochemicals effective on the various diseases or skin conditioning. Anti-inflammation, anti-atopic dermatitis, anti-melanogenesis, and collagen 
regeneration effects have been demonstrated in previous research [5-8]. Now, C. asiatica has become the most popular herb used as a cosmeceutical ingredient.

Syzygium formosum (Wall.) Masam leaf gained attention recently for its strong antiallergic function in a mouse food allergy model [9]. The plant generally grows in subtropical area of South Asia. In Vietnam, S. formosum has been used as a traditional remedy to treat atopic dermatitis or gastritis. Its leaves are used as a tea: leaves of $S$. formosum are dried, then boiled with water. A relatively large amount of S. formosum tea is recommended to treat allergic or inflammatory diseases [9]. A recent study revealed that $S$. formosum leaf contains a large amount of triterpenic acids such as asiatic acid, corosolic acid, and betulinic acid [10]. There are many other phytochemicals, but those triterpenic acids that are already well investigated should be the major functional phytochemicals. Previous research had scientifically proven the value of $S$. formosum as a functional ingredient for food and cosmetic industries. However, incomplete information on the variations of phytochemicals by individual trees can hinder industrial applications of the tree. Moreover, standardized cell-basis assays are essential to evaluate $S$. formosum as a cosmeceutical ingredient.

Skin aging is a natural process but could be greatly affected by exposure of skin to sunlight. Ultraviolet (UV) is one of the most harmful types of radiation and induces dramatic photoaging that is accompanied by wrinkles, strong pigmentation, and even skin cancers. UVB (280-320 $\mathrm{nm}$ of wave length) is $5-10 \%$ of sunlight UV, but UVB exposure may be the reason of serious skin damages by oxidation of lipids and proteins and further inflammatory cascades $[2,11]$. A human dermal keratinocyte, HaCaT, has been used to evaluate UVB effects on human skin cell, even though the cell line has dysfunctional p53 and shows subtle differences when compared with primary human dermal keratinocyte to the UV radiation $[12,13]$.

In this study, 12 wild trees of $S$. formosum were collected to investigate variations of triterpenic acids (TA). The leaves were dried and extracted with $70 \%$ ethanol. TA compositions were compared with that in C. asiatica, and functionalities of S. formosum leaf extract (SFLE) were evaluated by using a human keratinocyte, HaCaT cells. To induce inflammatory stress on the cells, UVB irradiation was used. Our study demonstrated potentials of the $S$. formosum leaf as a distinctive cosmeceutical ingredient with strong biological activities on skin.

\section{Results}

\subsection{Triterpenic Acid Compositions of 12 Wild S. formosum Trees}

More than 30 wild trees of $S$. formosum were found in Hanoi and the suburbs of Hanoi, Vietnam, which is a typical subtropical area in South-East Asia. The young trees had relatively small leaves which were not proper to harvest, therefore, we selected trees older than 5 years and harvested at early morning to noon. The trees had very large leaves, up to $40 \mathrm{~cm}$ long (Figure 1). Old trees had thicker and bigger leaves than young trees. The color of the leaves does not change by seasons in the growth area. 
A)

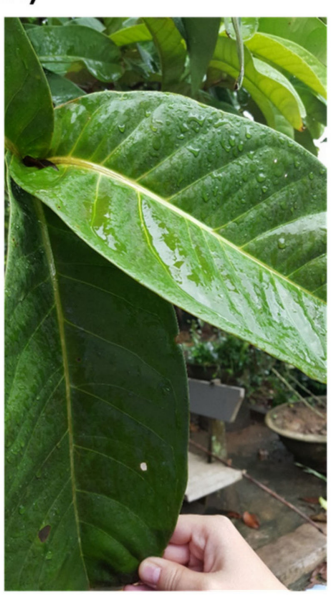

B)

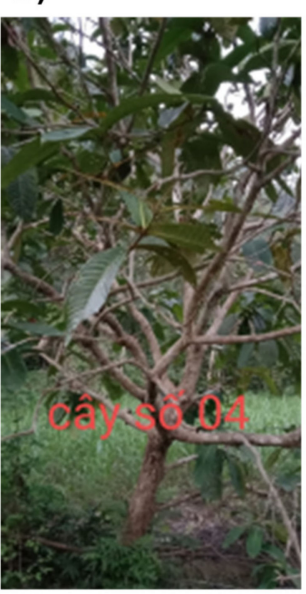

C)

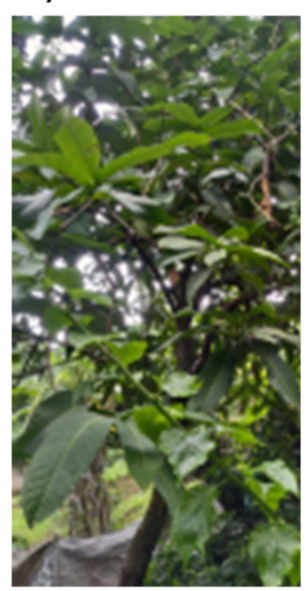

D)

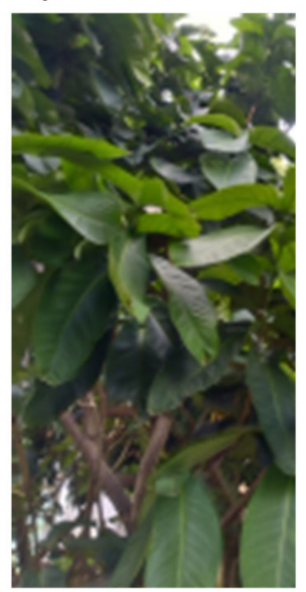

Figure 1. Examples of $S$. formosum leaf and trees. The size of the matured leaf could be estimated by comparison with a hand (A). Photos were taken just before harvesting leaves of each tree: (B) of S4, (C) of S10, and (D) of S12.

\subsubsection{Variations of Triterpenic Acids}

The twelve wild trees of S. formosum contained a significant amount of TAs in their leaves. Seven TAs including madecassic acid, asiatic acid, maslinic acid, colosolic acid, betulinic acid, oleanolic acid, and ursolic acid are commonly detected in the 12 trees (Table 1). Asiatic acid was the most present of the TAs, representing nearly a half of the total TA composition in some trees such as S4 and S11. The total amount of TAs varied by trees significantly, and showed more than 3-fold differences when minimal (S7) and maximal (S12) were compared. Either betulinic acid or colosolic acid was the second most represented TA in 12 trees of $S$. formosum. Others were relatively minor in the TA composition. Intriguingly, the total amount of TAs in $S$. formosum leaves was much greater than that of the C. asiatica. S12 had at least 5-fold greater amount of the total TAs than the C. asiatica (Table 1).

Table 1. Triterpenic acids in twelve Syzygium formosum trees. Some minor components that were close to the limits of quantification were excluded. For the comparison, two samples of Centella asiatica were analyzed by the same method.

\begin{tabular}{|c|c|c|c|c|c|c|c|c|}
\hline \multirow{2}{*}{ S. formosum } & \multicolumn{7}{|c|}{ Triterpenic Acids a (mg/g Dry Weight) } & \multirow{2}{*}{ Total } \\
\hline & $\begin{array}{l}\text { Madecassic } \\
\text { Acid }\end{array}$ & Asiatic Acid & $\begin{array}{l}\text { Maslinic } \\
\text { Acid }\end{array}$ & $\begin{array}{l}\text { Corosolic } \\
\text { Acid }\end{array}$ & $\begin{array}{l}\text { Betulinic } \\
\text { Acid }\end{array}$ & $\begin{array}{l}\text { Oleanolic } \\
\text { Acid }\end{array}$ & $\begin{array}{l}\text { Ursolic } \\
\text { Acid }\end{array}$ & \\
\hline S1 & $0.20 \pm 0.02$ & $3.36 \pm 0.51$ & $0.26 \pm 0.01$ & $0.89 \pm 0.15$ & $4.74 \pm 0.84$ & $0.065 \pm 0.005$ & $0.18 \pm 0.01$ & $9.695 \pm 1.545$ \\
\hline S2 & $0.67 \pm 0.01$ & $5.26 \pm 0.25$ & $0.53 \pm 0.02$ & $1.59 \pm 0.12$ & $3.09 \pm 0.02$ & $0.10 \pm 0.01$ & $0.23 \pm 0.01$ & $11.44 \pm 0.39$ \\
\hline S3 & $0.50 \pm 0.01$ & $5.57 \pm 0.17$ & $0.41 \pm 0.01$ & $1.25 \pm 0.06$ & $4.54 \pm 0.45$ & $0.12 \pm 0.01$ & $0.28 \pm 0.01$ & $12.65 \pm 0.68$ \\
\hline S4 & $0.98 \pm 0.04$ & $12.6 \pm 0.5$ & $1.04 \pm 0.05$ & $3.54 \pm 0.04$ & $5.27 \pm 0.36$ & $0.33 \pm 0.2$ & $0.81 \pm 0.04$ & $24.56 \pm 1.05$ \\
\hline S5 & $0.37 \pm 0.02$ & $5.89 \pm 0.02$ & $0.98 \pm 0.01$ & $3.76 \pm 0.12$ & $3.89 \pm 0.02$ & $0.26 \pm 0.01$ & $0.66 \pm 0.01$ & $15.81 \pm 0.18$ \\
\hline S6 & $0.25 \pm 0.04$ & $2.78 \pm 0.29$ & $0.44 \pm 0.04$ & $1.64 \pm 0.22$ & $4.23 \pm 0.43$ & $0.065 \pm 0.005$ & $0.16 \pm 0.01$ & $9.56 \pm 1.03$ \\
\hline S7 & $0.24 \pm 0.02$ & $3.99 \pm 0.05$ & $0.37 \pm 0.04$ & $1.35 \pm 0.07$ & $2.53 \pm 0.24$ & $0.08 \pm 0.01$ & $0.23 \pm 0.02$ & $8.77 \pm 0.43$ \\
\hline S8 & $0.44 \pm 0.03$ & $4.68 \pm 0.54$ & $0.84 \pm 0.06$ & $3.78 \pm 0.42$ & $5.11 \pm 0.50$ & $0.18 \pm 0.01$ & $0.52 \pm 0.05$ & $15.53 \pm 1.59$ \\
\hline S9 & $0.29 \pm 0.01$ & $6.81 \pm 0.46$ & $0.95 \pm 0.03$ & $3.83 \pm 0.30$ & $3.07 \pm 0.45$ & $0.22 \pm 0.02$ & $0.66 \pm 0.03$ & $15.81 \pm 1.18$ \\
\hline S10 & $0.39 \pm 0.03$ & $4.51 \pm 0.80$ & $0.84 \pm 0.05$ & $4.02 \pm 0.70$ & $5.89 \pm 0.78$ & $0.19 \pm 0.02$ & $0.60 \pm 0.03$ & $16.41 \pm 2.38$ \\
\hline S11 & $0.27 \pm 0.02$ & $7.76 \pm 0.61$ & $0.86 \pm 0.01$ & $3.66 \pm 0.47$ & $2.70 \pm 0.25$ & $0.19 \pm 0.02$ & $0.56 \pm 0.01$ & $15.99 \pm 1.35$ \\
\hline $\mathrm{S} 12$ & $0.82 \pm 0.01$ & $10.07 \pm 0.09$ & $2.16 \pm 0.02$ & $6.21 \pm 0.01$ & $6.05 \pm 0.22$ & $0.53 \pm 0.03$ & $1.36 \pm 0.02$ & $27.19 \pm 0.26$ \\
\hline Average & $0.42 \pm 0.25$ & $5.84 \pm 2.81$ & $0.79 \pm 0.47$ & $2.95 \pm 1.50$ & $4.24 \pm 1.20$ & $0.19 \pm 0.12$ & $0.51 \pm 0.32$ & $14.94 \pm 5.50$ \\
\hline C. asiatica & $1.72 \pm 0.26$ & $3.21 \pm 0.7$ & $\mathrm{NA}^{\mathrm{b}}$ & NA & NA & NA & NA & $4.94 \pm 0.96$ \\
\hline
\end{tabular}

${ }^{a}$ The order of chemicals follows retention times of triterpenic acids in HPLC-MS/MS analyses. ${ }^{b}$ Not available: the chemical was not detected or under the limits of quantification. 
In the anabolism of TAs, 2,3-oxidoscualene needs to be catalyzed into precursors of TAs such as amyrins ( $\alpha$ - and $\beta$-amyrin), lupeol, dammaranediol, lanosterol, or cycloartenol. The seven TAs found in the $S$. formosum trees are categorized into three groups according to their metabolic pathway in plants (Figure 2). Ursolic acid, corosolic acid, Asiatic acid, and madecassic acid are synthesized from $\alpha$-amyrin as a common precursor. $\beta$-amyrin derivatives include oleanolic acid and maslinic acid. Betulinic acid was the only TA which has lupeol as a precursor [14]. No derivatives from dammaranediol, lanosterol, or cycloartenol were detected from the $S$. formosum leaf extracts. It was notable that $\alpha$-Amyrin derivatives were major TAs in all the 12 trees.

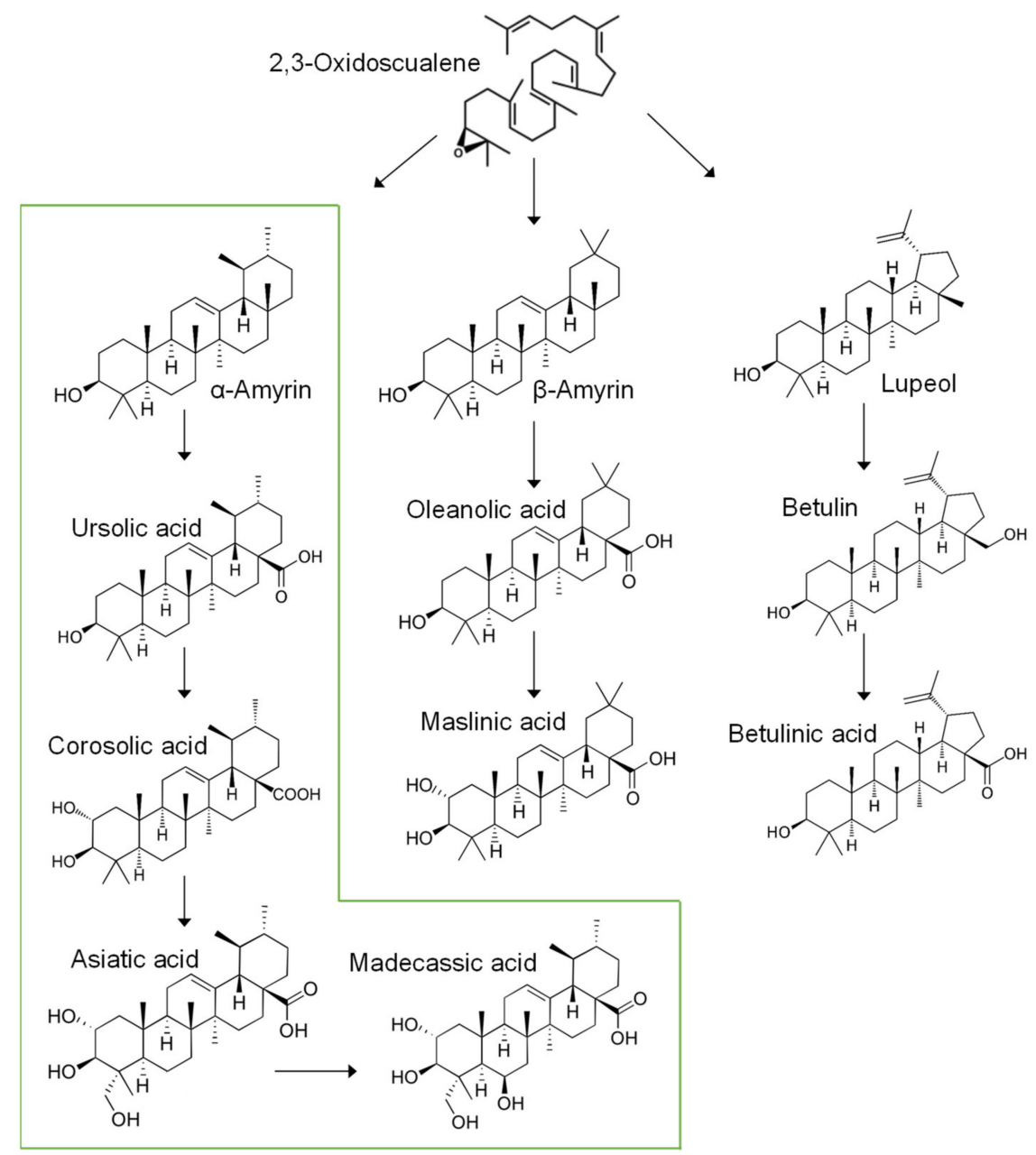

Figure 2. Metabolic pathways of triterpenic acids in Syzigium formosum. Metabolites which were detected in this study were included. The major metabolic pathway that has $\alpha$-amyrin as a precursor is boxed in green.

\subsubsection{Comparisons of Triterpenic Acids with Centella asiatica}

Twelve trees of $S$. formosum accumulated at least seven different TAs, while $C$. asiatica contained only two Tas, asiatic acid and madecassic acid. $\alpha$-Amyrin derivatives were more than $50 \%$ of the total Tas, but the $\beta$-amyrin pathway was very minor (Figure 3). Intriguingly, S. formosum leaves synthesized significant amount of betulinic acid with betulin which, was less than $5 \%$ of betulinic acid (data not shown).

As shown in Table 1, S. formosum accumulated madecassic acid, but it was only 5-10\% of asiatic acid. In contrast, madecassic acid amount in the C. asiatica reached more than $50 \%$ of asiatic acid. 

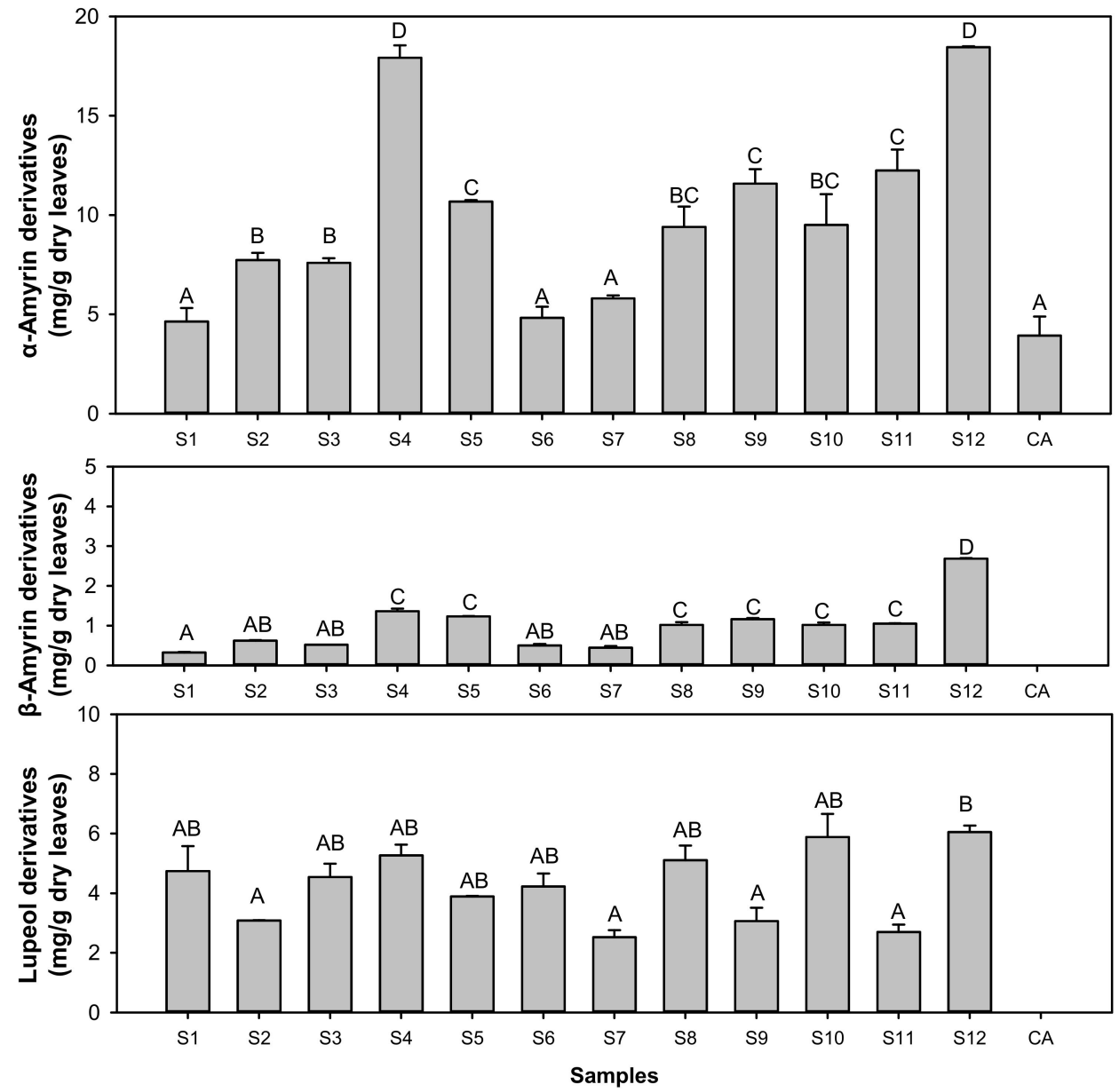

Figure 3. Comparison of TA amount grouped by metabolic pathways in the plant. S1-S12, Syzygium formosum samples; CA1 and 2, Centella asiatica samples. Note, no metabolite from $\beta$-amyrin and lupeol was detected in C. asiatica. Bars denoted with different alphabet are statistically different with $p$-value $<0.05$

\subsection{Antioxidative Activities and Total Phenolics}

Based on the triterpenic acid amount, S4 and S12 trees were selected for further chemical and biological examinations. Before cell-based assays, antioxidative power and total phenolic compounds of S4 and S12 were determined (Figure 4). The S. formosum samples had about 4-fold higher antioxidant levels than the C. asiatica. S4 and S12 exhibited a similar level of antioxidative compounds, but $\mathrm{S} 4$ had a significantly larger amount of the total phenolics than S12. It is notable that the total phenolics of S12 was more than 6-fold higher than that of the C. asiatica sample. 
A)

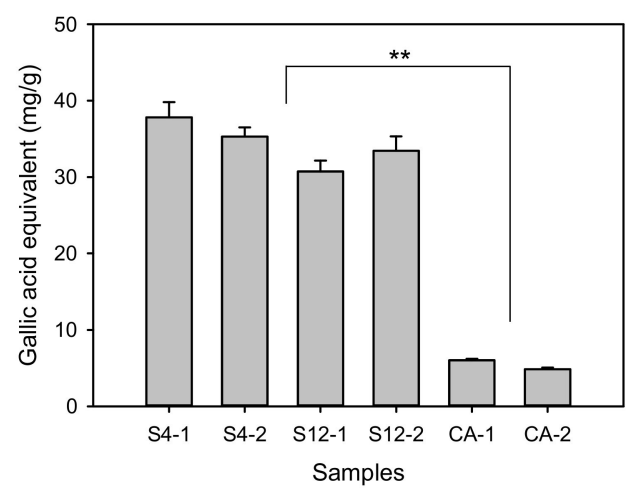

B)

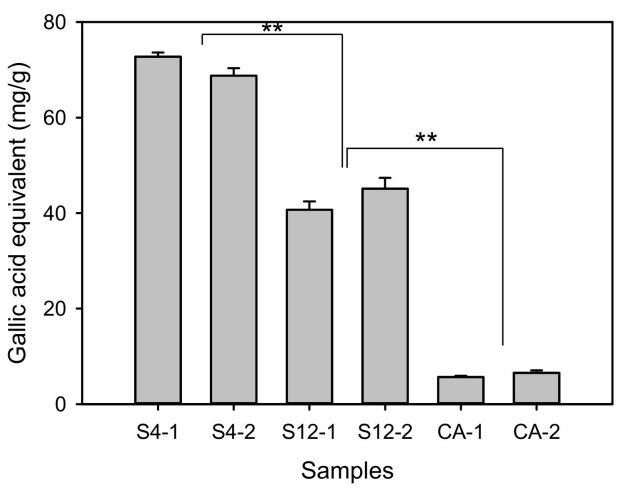

Figure 4. Antioxidative power (A) and total phenolics (B) of the plant extracts are expressed with gallic acid equivalent amount. S4 and S12 were the selected elite trees which accumulated the greatest amount of triterpenic acids of Syzygium formosum samples, and CA indicates the Centella asiatica. Double asterisks $\left.{ }^{* *}\right)$ indicate statistical difference $(p<0.01)$. Two independently prepared extracts were numbered in addition to the sample code. The assays using each extract were conducted in triplicate.

\subsection{Anti-Inflammatory Effects}

\subsection{1. mRNA Expression of Pro-Inflammatory Genes}

Anti-inflammatory effects of the S. formosum were assessed using UVB induced inflammation model of HaCaT. Based on the cytotoxicity evaluations with MTT assay (data not shown), $5 \mu \mathrm{g} / \mathrm{mL}$ concentration was selected for the biological activity analyses. UVB radiation greatly enhanced pro-inflammatory responses such as IL-6, IL-8, and cyclooxygenase-2 of HaCaT, but IL-1 $\beta$ level was not much increased. Both the $S$. formosum and the C. asiatica samples significantly repressed IL-1 $\beta$ and COX-2 expressions in the HaCaT cells that were exposed to UVB to induce inflammatory responses (Figure 5A,D). The mRNA levels of IL-6 and 8 were greatly reduced in S4 and S12 samples, while the extracts from C. asiatica did not produce significant changes (Figure 5B,C).

A)
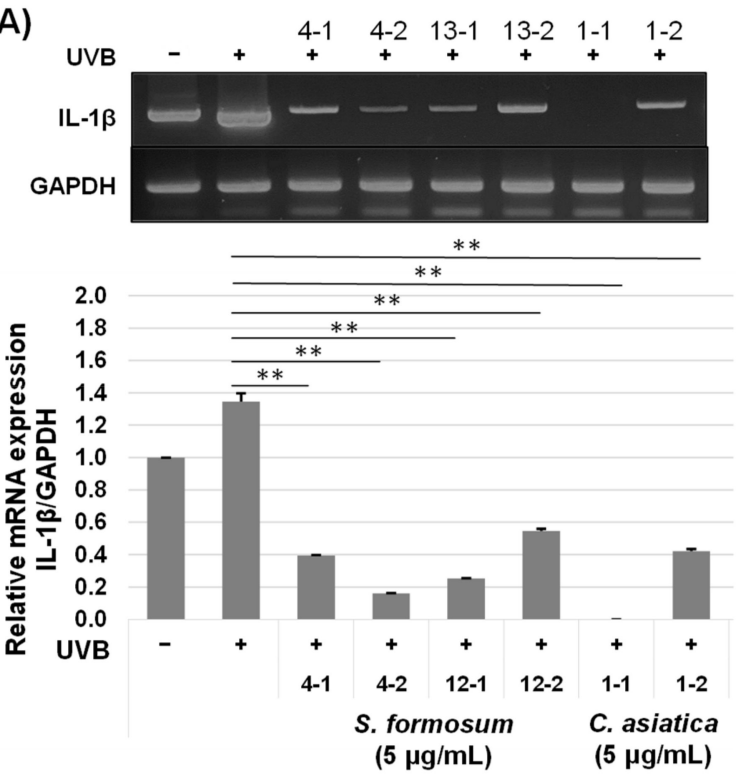

B)
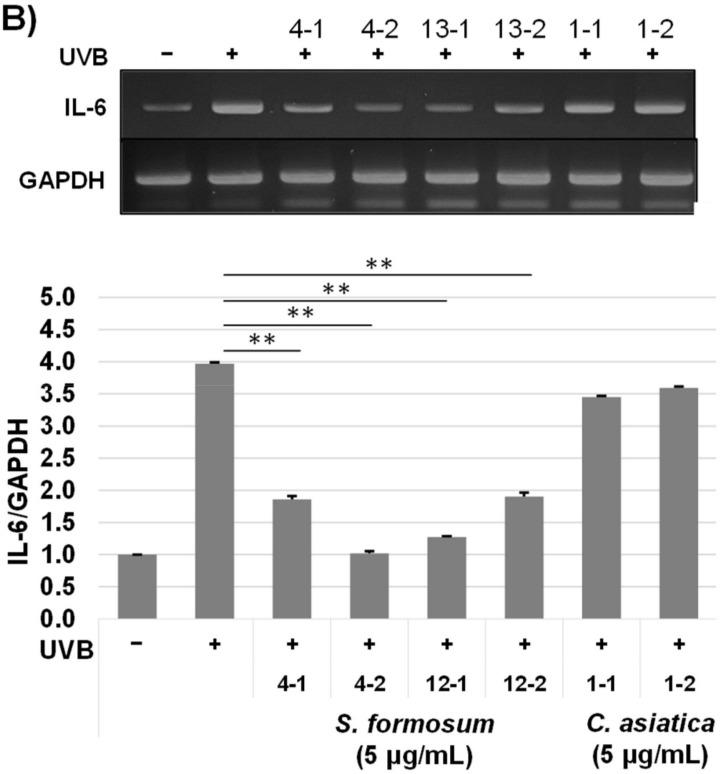

Figure 5. Cont. 

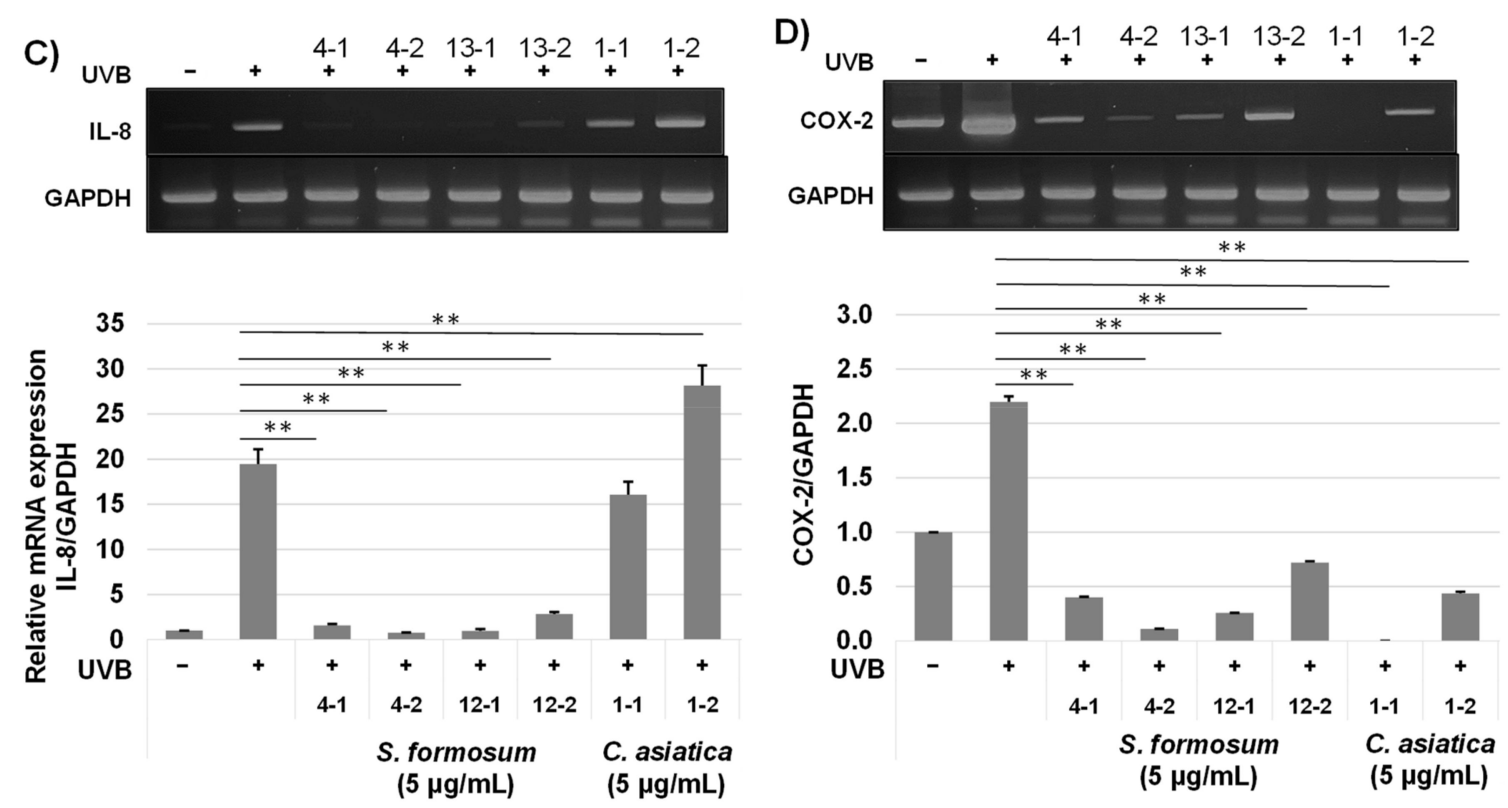

Figure 5. Repression of pro-inflammatory genes by Syzygium formosum extracts was estimated by mRNA expression. The extracts of S4 and S12 were selected based on the total triterpenic acid and compared with the Centella asiatica extract. The cells were exposed to $20 \mathrm{~mJ} / \mathrm{cm}^{2}$ of UVB. IL-1 $\beta$ (A), IL-6 (B), IL-8 (C), and COX-2 (D) expressions were compared in relative amounts to a house keeping gene, glyceraldehyde 3-phosphate dehydrogenase (GAPDH). Double asterisks $\left.{ }^{(*}\right)$ indicate statistical difference $(p<0.01)$. Two independently prepared extracts were numbered in addition to the sample code. The assays using each extract were conducted in triplicate.

The extracts from the same sample showed slight variation, but the difference was not so great to affect the interpretation of the results. The results indicate that $S$. formosum has great anti-inflammatory effects on the UVB radiated skin cells than C. asiatica.

\subsubsection{Protein Levels of Pro-Inflammatory Cytokines}

To estimate anti-inflammatory effects of $S$. formosum, cytokine concentrations of IL- $1 \beta$, IL-6 and IL-8 in HaCaT cell culture medium were analyzed. Although the mRNA level of IL-1 $\beta$ was not greatly increased (Figure 5A), all the cytokines in protein level were increased profoundly by UVB radiation. The extracts from $\mathrm{S} 4$ and $\mathrm{S} 12$ greatly reduced concentrations of IL-1 $\beta$, IL-6, and IL-8 in the culture medium (Figure 6). Intriguingly, the C. asiatica sample also showed repressions of all the three cytokines. However, the anti-inflammatory effects of the $S$. formosum were significantly higher than that of the $C$. asiatica. 

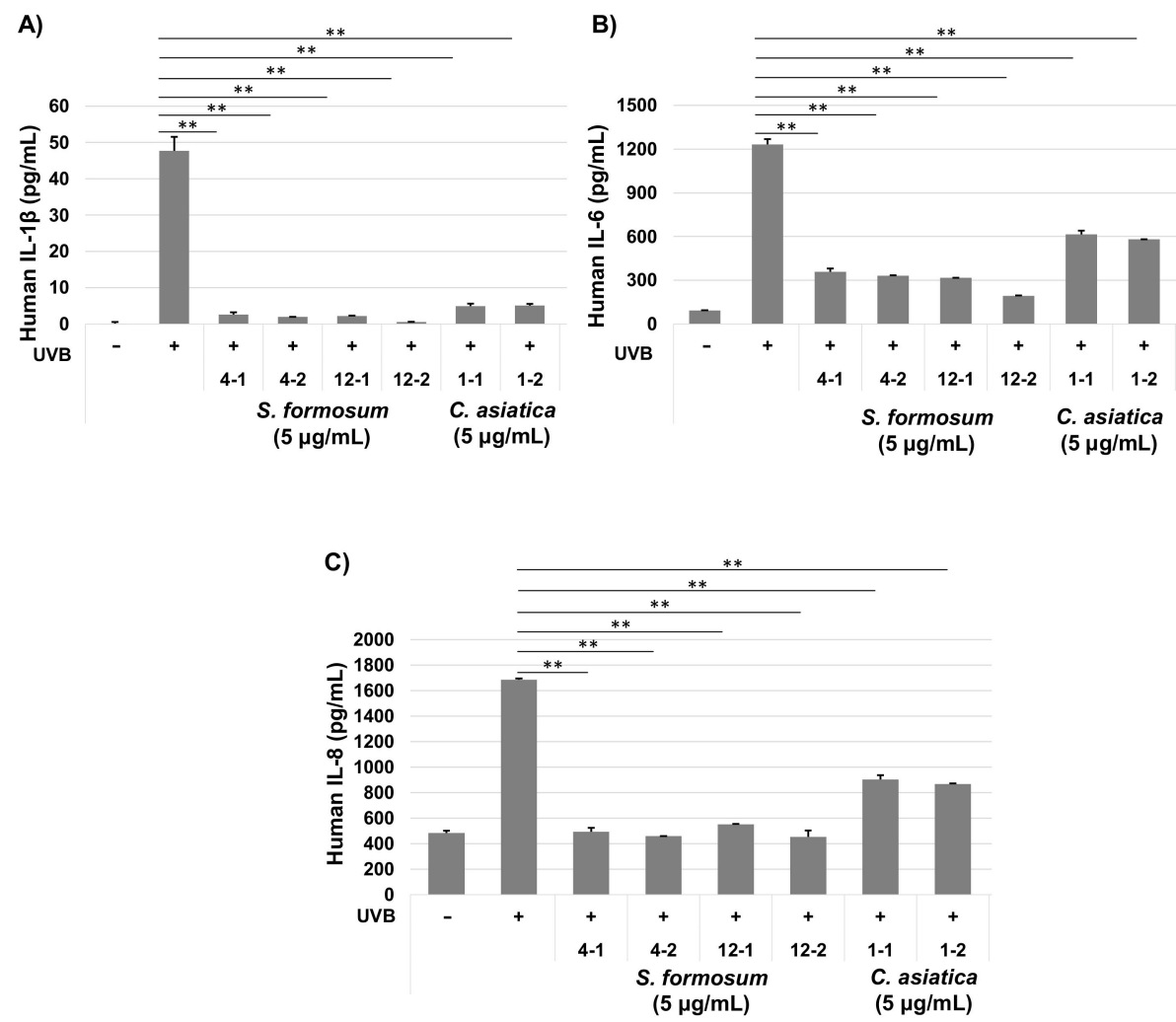

Figure 6. Protein levels of pro-inflammatory cytokines, IL-1ß (A), IL-6 (B), and IL-8 (C) after a Syzigium formosum extract treatment. The extracts of S4 and S12 were selected based on the total triterpenic acid and compared with the Centella asiatica extract. The cells were exposed to $20 \mathrm{~mJ} / \mathrm{cm}^{2}$ of UVB. Double asterisks $\left({ }^{* *}\right)$ indicate statistical difference $(p<0.01)$. Two independently prepared extracts were numbered in addition to the sample code. The assays using each extract were conducted in triplicate.

\section{Discussion}

In this study, 12 wild trees of $S$. formosum were analyzed to provide valuable information for industrial uses of the tree. This subtropical tree has very large and relatively thick leaves that contains large amounts of secondary metabolites. Among various phytochemicals, TAs were considered as major products based on the previous research on the chemical composition of $S$. formosum leaves, and many reports on the biological activities of TAs are available $[7,9,15,16]$. It is well known that secondary metabolites are affected by environmental factors as well as genetic backgrounds. Nutrients, sunlight, and temperature are the major environmental factors which manipulate the metabolism of phytochemicals [14]. In this study, wild trees in the Hanoi area were selected. Therefore, variations of temperature and sunlight might be minor. The nutrients, however, possibly affected the metabolism of $S$. formosum. In further study, we will evaluate the effects of environmental factors on the metabolism of secondary metabolites in S. formosum.

Initially, we found $S$. formosum leaves accumulated various TAs that are derivatives of amyrins and lupeol. As expected, lanosterol and its derivatives that are common precursors of sterol synthesis in animals and fungi were not detected in the extracts of S. formosum. Similar to C. asiatica, the most abundant TA was asiatic acid in all the 12 trees. The Asiatic acid slightly exceeded 50\% of the total TAs in S4. The asiatic acid amount in dried leaves was significantly greater in the S. formosum samples than the C. asiatica. Madecassic acid, which is synthesized from asiatic acid in plants, was detected and quantified in this study, but previous studies reported no detection [10]. It is notable that glycosylated Tas were hardly detected in the S. formosum leaf. Harvesting conditions, such as different seasons, may affect the aglycon and glycoside composition, but this question should be answered 
by further studies. Among the 12 trees, S4 and S12 accumulated much greater amounts of TAs than others. Moreover, total TAs in S4 and S12 were at least 5-fold higher than the C. asiatica. Taken together, S4 and S12 were determined as elite trees which could be used for breeding of $S$. formosum in the near future.

To our knowledge, a genetic study of $S$. formosum has not been conducted yet. But our results indicate that triterpene anabolism is very strong in $S$. formosum leaves and favors an $\alpha$-amyrin pathway. $\beta$-Amyrin, however, exhibited a very low proportion in the TA composition. Corosolic acid, which is an intermediate $\alpha$-amyrin pathway to asiatic acid and betulinic acid, was relatively high in all the 12 trees. Therefore, similar to a previous report, asiatic acid, betulinic acid, and corosolic acid could be considered as standard components in the extract of $S$. formosum leaf. When combined with genetic studies, we can develop a proper strategy to control and enhance production of these secondary metabolites in S. formosum.

Many triterpenic acids are proved as strong bioactive compounds and used as pharmaceutical and cosmeceutical ingredients. Asiatic acid, madecassic acid, corosolic acid, and betulinic acids are representative chemicals that are well investigated and industrialized in those ways [16-19]. In this study, HaCaT cells radiated with UVB were adopted due to the well-established references of inflammatory responses of HaCaT to UVB $[12,13]$. Although $\mathrm{HaCaT}$ has dysfunctional p53, the cell shows similar inflammation responses to primary human keratinocyte. UVB radiation induces various stress responses including pro-inflammatory cytokine expressions. In a previous report, among pro-inflammatory cytokines, tumor necrosis factor- $\alpha$ (TNF- $\alpha$ ) did not respond strongly when HaCaT was exposed to weak UVB [13]. This phenomenon was also found in our study (data not shown), and basal levels of pro-inflammatory cytokines in $\mathrm{HaCaT}$ were relatively high.

The results shown in Figures 5 and 6 clearly indicate that extract of S. formosum leaf can repress or stop the inflammation cascade in skin induced by UVB radiation. Low concentration of $S$. formosum leaf extracts reduced IL-1 $\beta$, IL-6, and IL-8 at a similar level to the control group, which showed basal level of cytokines. IL-1 $\beta$ is one of the major cytokines produced by stratum corneum and fibroblast [20,21]. It activates an inflammatory response of epithelial cells and induces differentiation of Th17. IL-6 has a major role in the acute inflammatory response of the liver. In human skin, a high level of IL-6 induces fibrosis by complex interplay with TFG- $\beta$ in dermal cells [22]. IL-8 is produced mainly by macrophage, epithelial cells, and airway smooth muscle cells. This cytokine is a neutrophil chemotactic factor and stimulates phagocytic functions of that immune cell. In a recent study, IL-1 $\beta$ and IL-8 were found to be highly expressed cytokines in the stratum corneum of atopic dermatitis lesional skin [21]. In addition, the level of IL-1 $\beta$ showed a positive correlation with severity of the disease. Increased expression of COX-2 can induce premature aging of a transgenic mouse model [23].

The S. formosum samples contained various components as well as seven TAs [10], therefore, anti-inflammatory functions of the extract were derived from the complex of the phytochemicals. However, it is plausible that the TAs are the major functional components due to their high concentration in the extract. The total TAs in the extract were around 15\% (S4) to $25 \%$ (S12) of total dry matter content of the extract. The major TA in S. formosum, asiatic acid, is well recognized by its strong anti-oxidative, anti-inflammatory, and antiallergic effects. Corosolic acid inhibits inflammation of adipose tissue in a high-fat diet mouse model. Recently, protecting effects of corosolic acid on the diabetic renal damage were reported [17]. One of the major TAs in the S. formosum leaf, betulinic acid, also has potent anti-inflammatory and anti-cancer effects. In a lethal endodoxemia mouse model, feeding of betulinic acid significantly decreased TNF- $\alpha$ and increased IL-10 [24]. Recently, anti-cancer functions of betulinic acid on the glioblastoma were reported [18]. Regarding contents of TAs, S. formosum leaf is a good raw material to provide the biological benefits of asiatic acid, corosolic acid, and betulinic acid to human health.

Taken all together, we have shown that $S$. formosum leaves contained greater compositions of biologically active phytochemicals. The extract of $S$. formosum leaves showed 
stronger anti-inflammatory effects than C. asiatica on human keratinocyte, HaCaT. In further studies, biological activities on the skin of $S$. formosum leaves will be conducted to focus on anti-wrinkle activities and improvements of skin barrier functions.

\section{Materials and Methods}

\subsection{Plant Samples and Reagents}

Leaves of S. formosum were harvested and sun-dried in September-October, 2020. Wild trees older than 5 years were selected in the Hanoi area, Vietnam. Dried C. asiatica from Indonesia was purchased online (Jung Woo-dang, Seoul, Korea). Cell culture reagent, Dulbecco Modified Eagle Medium (DMEM), was purchased from WELGENE (Daegu, Korea). Penicillin, streptomycin, and $0.05 \%$ trypsin-EDTA were purchased from Gibco (Waltham, MA, USA). Reverse transcription mix (5x master premix, Elpis-Biotech, Daejeon, Korea) and Taq PCR premix (AccuPower ProFi, Bioneer, Daejeon, Korea) were used for gene works. HPLC analysis grade asiatic acid, madecassic acid, corosolic acid, betulinic acid, oleanolic acid (4 chemicals: Sigma-Aldrich, St. Louis, MO, USA), ursolic acid (Tokyo Chemical Industry, Tokyo, Japan), and maslinic acid (Chengdu Biopurify Phytochemicals Ltd., Chengdu, China) with at least $95 \%$ purity were used.

\subsection{Extraction of Plants}

Dried leaves of $S$. formosum or C. asiatica containing 6-11\% water were ground using a home blender. Then, $2 \mathrm{~g}$ of each sample was mixed with $40 \mathrm{~mL}$ of $70 \%$ ethanol at $50{ }^{\circ} \mathrm{C}$ for $24 \mathrm{~h}$. The supernatant was separated and harvested from insoluble matters by centrifugation at $4{ }^{\circ} \mathrm{C}(12,000 \times g)$ for $20 \mathrm{~min}$. The extracts were freeze-dried to powder and kept at $-18^{\circ} \mathrm{C}$ before use.

\subsection{Instrumental Analyses of Triterpenic Acids}

Sample pretreatment for liquid chromatography-mass spectrometry (LC-MS) was conducted using solid-phase extraction (C18 cartridge, $200 \mathrm{mg}, 3 \mathrm{~mL}$, Waters, Milford, MA, USA) to remove hydrophilic components, such as sugars. Recovery of this was $90-105 \%$. UPLC system (Agilent 1290 Infinity, Agilent Technologies Inc., Santa Clara, CA, USA) with a triple quadrupole mass spectrometer (Agilent 6470, Agilent Technologies Inc.), equipped with a turbo ion spray source was used for the identification and quantification of TAs. C18 column $(2.1 \times 100$ mm, $1.8 \mu \mathrm{m}$, Zorbax Eclipse Rapid Resolution High Definition, Agilent Technologies Inc.) was stabilized with $5 \mathrm{mM}$ ammonium formate (solvent A), and analytes were eluted with a mixture of $95 \%$ methanol and 5\% isopropyl alcohol with $5 \mathrm{mM}$ ammonium formate (solvent $\mathrm{B}$ ). The flow rate was $0.2 \mathrm{~mL} / \mathrm{min}$ with gradient of solvent $\mathrm{B}$ : 0-1 $\mathrm{min}, 70 \%$; $1-3 \mathrm{~min}, 80 \%$; 3-8 $\mathrm{min}, 82 \%$; $8-20 \mathrm{~min}, 84 \%$; and $20-25.5 \mathrm{~min}, 100 \%$. The gas flow and temperature were $10 \mathrm{~L} / \mathrm{min}$ and $270{ }^{\circ} \mathrm{C}$, respectively. The nebulizer was set as $40 \mathrm{psi}$, and the sheath gas temperatures was $300^{\circ} \mathrm{C}$. Electrospray ionization with voltage of $3500 \mathrm{~V}$ was used with the positive mode. Multiple reaction monitoring (MRM) was adapted for quantification of TAs. Seven standard molecules were analyzed by the same method including solid-phase extraction.

\subsection{Chemical and Biological Activity Assay \\ 4.4.1. Antioxidative Activity}

Reducing power of the plant extracts was determined by DPPH method. The assay was conducted as described previously [25]. Briefly, $0.95 \mathrm{~mL}$ of $0.2 \mathrm{mM}$ DPPH solution was mixed with $0.05 \mathrm{~mL}$ of the plant extract which was diluted appropriately and incubated in the dark and at room temperature for $30 \mathrm{~min}$. Absorbance of the reacted sample was measured at $517 \mathrm{~nm}$. In this study, gallic acid was used as a standard molecule.

\subsubsection{Total Phenolic Compounds}

Total phenolic compounds were determined by the Folin-Ciocalteu reaction method. Overall assay was conducted as described previously with slight modification [26]. First, 
the plant extracts were properly diluted. The diluted sample $0.1 \mathrm{~mL}$, distilled water $1.5 \mathrm{~mL}$, and the Folin-Ciocalteu solution were mixed and incubated for $5 \mathrm{~min}$. After adding $20 \%$ sodium carbonate $0.3 \mathrm{~mL}$, the mixture stood at room temperature for $30 \mathrm{~min}$. Then, absorbance at $765 \mathrm{~nm}$ was measured. Gallic acid was used as a standard material.

\subsubsection{Cell Culture and Viability Assay}

The HaCaT cell line was kindly provided by Dr. J.M. Shin at Research Institute for Medical Science, College of Medicine, Chungnam National University, Korea. Cytotoxicity of the plant extract was evaluated with 3-(4,5-dimethylthiazol-2-yl)-2,5-diphenyltetrazolium bromide (MTT; Sigma-Aldrich Co., St Louis, Mo, USA) assay. Briefly, the cells $\left(0.2 \times 10^{5} / \mathrm{mL}\right)$ were seeded on a 96-well plate and cultured overnight. The plant extract was first dissolved in DMSO and adjusted with the culture medium to the final concentration. The properly diluted DMSO with the culture medium, as a vehicle, was mixed into the culture plate. After $24 \mathrm{~h}$ incubation, the MTT solution was added and incubated for a further $3 \mathrm{~h}$. The supernatant was removed, then $0.1 \mathrm{~mL}$ of DMSO was added. Finally, the absorbance at 540 nm was measured with microplate reader (Epoch, Biotek, Winooski, VT, USA).

\subsubsection{Anti-Inflammatory Effects of Plant Extracts}

Firstly, the UVB radiation condition was optimized to induce pro-inflammatory cytokines (IL-1 $\beta$, IL-6, and IL-8) and COX-2. A range of UVB radiation, $5-40 \mathrm{~mJ} / \mathrm{cm}^{2}$, was tested and $20 \mathrm{~mJ} / \mathrm{cm}^{2}$ was selected for further experiment condition.

HaCaT cells $\left(1.0 \times 10^{6} / \mathrm{mL}\right)$ were seeded in a 6-well plate and incubated overnight. Six hours before UVB exposure, the plant extract was treated on the cells at the final concentration of $5 \mu \mathrm{g} / \mathrm{mL}$. After removing medium, $2 \mathrm{~mL}$ serum free-media (DMEM), having the same concentration of the plant extract, was added to the cells. The cells were incubated for $18 \mathrm{~h}$, then harvested for total RNA extraction. The cDNA was prepared from the total RNA and used for the PCR to determine mRNA expression of IL-1 $\beta$, IL-6, IL-8, and COX-2. The expression levels of the target genes were compared with a house keeping gene, actin. Sequences of PCR primers are shown in Table 2.

Table 2. PCR primers of pro-inflammatory genes and actin.

\begin{tabular}{ccc}
\hline Gene & Forward & Reverse \\
\hline IL-1 $\beta$ & GGG CCT CAA GGA AAA GAA TC & TTC TGC TTG AGA GGT GCT GA \\
\hline IL-6 & AGG AGA CTT GCC TGG TGA AA & CAG GGG TGG TTA TTG CAT CT \\
\hline IL-8 & TCT GTG TGA AGG TGC ACT T & AGC CCT CTT CAA AAA CTT CT \\
\hline COX-2 & GGT CTG GTG CCT GGT CTG ATG & GTC CTT TCA AGG AGA ATG GTG C \\
\hline Actin & GGC GGA CTA TGA CTT AGT TG & AAC AAC AAT GTG CAA TCA A \\
\hline
\end{tabular}

For the ELISA, the supernatants were collected, and the concentrations of cytokines correspondingly quantified. ELISA assays for IL-1 $\beta$, IL-6, and IL-8 were executed with a Thermo Fisher Scientific ELISA system (Thermo Fisher Scientific, Inc., Waltham, MA, USA) according to the protocols from the manufacturer.

\subsection{Statistical Analyses}

Statistical analyses between the control group and the plant extract treatment group were conducted using one-way ANOVA. The statistical significance was indicated by * for $p<0.05$ and ${ }^{* *}$ for $p<0.01$, respectively. 


\begin{abstract}
Author Contributions: Conceptualization, J.-T.P. and J.-I.P.; methodology, M.Y.K., J.-I.P., J.K. and J.-T.P.; software and validation, M.Y.K. and J.K.; formal analysis, H.-a.P. and J.L.; investigation, H.a.P., N.-Y.L. and K.-b.P.; resources, V.D.N. and C.-K.L.; data curation, J.K.; writing-original draft preparation, M.Y.K.; writing-review and editing, J.-T.P. and J.-I.P.; visualization, H.-a.P.; supervision, J.-T.P.; project administration, C.-K.L.; funding acquisition, C.-K.L. All authors have read and agreed to the published version of the manuscript.
\end{abstract}

Funding: This research was supported by a grant of the Korea Health Technology R\&D Project through the Korea Health Industry Development Institute (KHIDI), funded by the Ministry of Health \& Welfare, Republic of Korea (grant number: HP20C0211).

Institutional Review Board Statement: Not applicable.

Informed Consent Statement: Not applicable.

Data Availability Statement: All the data supporting this article were included in the main text.

Conflicts of Interest: The authors declare no conflict of interest.

\title{
References
}

1. Chiocchio, I.; Mandrone, M.; Sanna, C.; Maxia, A.; Tacchini, M.; Poli, F. Screening of a hundred plant extracts as tyrosinase and elastase inhibitors, two enzymatic targets of cosmetic interest. Ind. Crop. Prod. 2018, 122, 498-505. [CrossRef]

2. Mukherjee, P.K.; Maity, N.; Nema, N.K.; Sarkar, B.K. Bioactive compounds from natural resources against skin aging. Phytomedicine 2011, 19, 64-73. [CrossRef] [PubMed]

3. Hashim, P.; Sidek, H.; Helan, M.H.M.; Sabery, A.; Palanisamy, U.D.; Ilham, M. Triterpene composition and bioactivities of Centella asiatica. Molecules 2011, 16, 1310-1322. [CrossRef] [PubMed]

4. Bylka, W.; Znajdek-Awiżeń, P.; Studzińska-Sroka, E.; Dańczak-Pazdrowska, A.; Brzezińska, M. Centella asiatica in dermatology: An overview. Phytother. Res. 2014, 28, 1117-1124. [CrossRef]

5. An, I.-S.; An, S.; Choe, T.-B.; Kang, S.-M.; Lee, J.H.; Park, I.-C.; Jin, Y.-W.; Lee, S.-J.; Bae, S. Centella asiatica protects against UVB-induced HaCaT keratinocyte damage through microRNA expression changes. Int. J. Mol. Med. 2012, 30, 1349-1356. [CrossRef]

6. Jung, E.; Lee, J.-A.; Shin, S.; Roh, K.-B.; Kim, J.-H.; Park, D. Madecassoside inhibits melanin synthesis by blocking ultravioletinduced inflammation. Molecules 2013, 18, 15724-15736. [CrossRef]

7. Kwon, K.J.; Bae, S.; Kim, K.; An, I.S.; Ahn, K.J.; An, S.; Cha, H.J. Asiaticoside, a component of Centella asiatica, inhibits melanogenesis in B16F10 mouse melanoma. Mol. Med. Rep. 2014, 10, 503-507. [CrossRef]

8. Lee, J.-H.; Kim, H.-L.; Lee, M.H.; You, K.E.; Kwon, B.-J.; Seo, H.J.; Park, J.-C. Asiaticoside enhances normal human skin cell migration, attachment and growth in vitro wound healing model. Phytomedicine 2012, 19, 1223-1227. [CrossRef]

9. Nguyen, T.M.N.; Lomunova, M.; Vu, T.P.D.; Le, B.V.; Kim, Y.H.; Kang, J.S.; Hwang, I. Anti-allergic effects of the ethanol extract of Syzygium formosum (Wall.) Masam leaves and its immunoregulatory mechanisms. J. Ethnopharmacol. 2018, 211, 171-179. [CrossRef]

10. Vu, T.P.D.; Khong, T.Q.; Nguyen, T.M.N.; Kim, Y.H.; Kang, J.S. Phytochemical profile of Syzygium formosum (Wall.) Masam leaves using HPLC-PDA-MS/MS and a simple HPLC-ELSD method for quality control. J. Pharm. Biomed. Anal. 2019, 168, 1-12.

11. Lee, Y.S.; Jin, D.-Q.; Beak, S.-M.; Lee, E.-S.; Kim, J.-A. Inhibition of ultraviolet-A-modulated signaling pathways by asiatic acid and ursolic acid in HaCaT human keratinocytes. Eur. J. Pharmacol. 2003, 476, 173-178.

12. Yoshizumi, M.; Nakamura, T.; Kato, M.; Ishioka, T.; Kozawa, K.; Wakamatsu, K.; Kimura, H. Release of cytokines/chemokines and cell death in UVB-irradiated human keratinocytes, HaCaT. Cell Biol. Int. 2008, 32, 1405-1411. [CrossRef]

13. Muthusamy, V.; Piva, T.J. A comparative study of UV-induced cell signalling pathways in human keratinocyte-derived cell lines. Arch. Dermatol. Res. 2013, 305, 817-833. [CrossRef]

14. Biswas, T.; Dwivedi, U.N. Plant triterpenoid saponins: Biosynthesis, in vitro production, and pharmacological relevance. Protoplasma 2019, 256, 1463-1486. [CrossRef]

15. Wu, Z.-W.; Li, W.-B.; Zhou, J.; Liu, X.; Wang, L.; Chen, B.; Wang, M.-K.; Ji, L.; Hu, W.-C.; Li, F. Oleanane-and ursane-type triterpene saponins from Centella asiatica exhibit neuroprotective effects. J. Agric. Food Chem. 2020, 68, 6977-6986. [CrossRef]

16. Sirignano, C.; Nadembega, P.; Poli, F.; Romano, B.; Lucariello, G.; Rigano, D.; Taglialatela-Scafati, O. Triterpenoids from Vitellaria paradoxa stem barks reduce nitrite levels in LPS-stimulated macrophages. Plants 2021, 10, 1006. [CrossRef]

17. Yang, J.; Leng, J.; Li, J.-J.; Tang, J.-f.; Li, Y.; Liu, B.-L.; Wen, X.-D. Corosolic acid inhibits adipose tissue inflammation and ameliorates insulin resistance via AMPK activation in high-fat fed mice. Phytomedicine 2016, 23, 181-190. [CrossRef]

18. Lo, W.-L.; Hsu, T.-I.; Yang, W.-B.; Kao, T.-J.; Wu, M.-H.; Huang, Y.-N.; Yeh, S.-H.; Chuang, J.-Y. Betulinic acid-mediated tuning of PERK/CHOP signaling by Sp1 inhibition as a novel therapeutic strategy for glioblastoma. Cancers 2020, 12, 981. [CrossRef]

19. Chianese, G.; Masi, F.; Cicia, D.; Ciceri, D.; Arpini, S.; Falzoni, M.; Pagano, E.; Taglialatela-Scafati, O. Isomadecassoside, a new ursane-type triterpene glycoside from Centella asiatica leaves, reduces nitrite levels in LPS-stimulated macrophages. Biomolecules 2021, 11, 494. [CrossRef]

20. Clausen, M.-L.; Kezic, S.; Olesen, C.; Agner, T. Cytokine concentration across the stratum corneum in atopic dermatitis and healthy controls. Sci. Rep. 2020, 10, 21895. [CrossRef] 
21. Lyubchenko, T.; Collins, H.K.; Goleva, E.; Leung, D.Y. Skin tape sampling technique identifies proinflammatory cytokines in atopic dermatitis skin. Ann. Allergy Asthma Immunol. 2021, 126, 46-53.e42. [CrossRef]

22. Johnson, B.Z.; Stevenson, A.W.; Prêle, C.M.; Fear, M.W.; Wood, F.M. The role of IL-6 in skin fibrosis and cutaneous wound healing. Biomedicines 2020, 8, 101. [CrossRef]

23. Kim, J.; Vaish, V.; Feng, M.; Field, K.; Chatzistamou, I.; Shim, M. Transgenic expression of cyclooxygenase-2 (COX2) causes premature aging phenotypes in mice. Aging 2016, 8, 2392. [CrossRef]

24. Costa, J.F.O.; Barbosa-Filho, J.M.; de Azevedo Maia, G.L.; Guimarães, E.T.; Meira, C.S.; Ribeiro-dos-Santos, R.; de Carvalho, L.C.P.; Soares, M.B.P. Potent anti-inflammatory activity of betulinic acid treatment in a model of lethal endotoxemia. Int. Immunopharmacol. 2014, 23, 469-474. [CrossRef]

25. Mensor, L.L.; Menezes, F.S.; Leitão, G.G.; Reis, A.S.; Santos, T.C.d.; Coube, C.S.; Leitão, S.G. Screening of Brazilian plant extracts for antioxidant activity by the use of DPPH free radical method. Phytother. Res. 2001, 15, 127-130. [CrossRef]

26. Kim, S.-J.; Cho, M.-H. Melatonin and polyphenol contents in some edible sprouts (alfalfa, chicory, rape, red kale and sunflower). Prev. Nutr. Food Sci. 2011, 16, 184-188. [CrossRef] 